\title{
THEY SHOULDN'T POST THAT! STUDENT PERCEPTION OF INAPPROPRIATE POSTS ON FACEBOOK REGARDING ALCOHOL CONSUMPTION AND THE IMPLICATIONS FOR PEER SOCIALIZATION
}

\author{
Loreen Wolfer \\ Department of Sociology, University of Scranton, Scranton, PA USA
}

Received 2014-07-07; Revised 2014-07-15; Accepted 2014-07-18

\begin{abstract}
Many students believe that drinking alcohol is part of the collegiate experience and showing that one consumes alcohol is an important part of establishing that one fits into this atmosphere. Facebook is one means in which college students present the appearance of social conformity in order to gain peer approval; yet, in accordance with Privacy Calculus Theory, students also need to weigh the potential cons of non-peer disapproval for drinking disclosures. However, for the process of Privacy Calculus theory to work with regards to peer acceptance, students need to obtain feedback from their peers in order to accurately assess the pros and cons of different levels of disclosure. Seven separate focus groups involving a total of 46 students at a small, private college in Pennsylvania were conducted to explore whether college students perceive limitations in appropriateness for Facebook drinking posts and, subsequently if they do anything to peers to express disapproval if this line is crossed. Findings suggest that college students consider Facebook posts about underage drunken behavior and about drunken vomiting inappropriate because non-peer Facebook friends, such as family, may see the posts; and, additionally, in the case of vomiting, because sharing such behavior is deemed unnecessary and excessive. However, findings also suggest that students ignore these inappropriate posts without offering any sanctioning comments to their peers and in many instances actually find these posts "entertaining" even though these students form negative opinions of the discloser. This questions how well Facebook works towards helping late adolescents understand the approved behaviors of their cohort and, in accordance with Privacy Calculus Theory, how accurately peers can evaluate the pros and cons of disclosure.
\end{abstract}

Keywords: College Students, Alcohol, Facebook, Peer Socialization

\section{INTRODUCTION}

Electronic forms of communication, such as email, texts, list-serves, Facebook and Twitter, shape adolescents today in new ways that adolescents twenty-five years ago did not experience. According to (Brenner, 2012), more than $80 \%$ of teens and young adults in the United States use Facebook; and, (Ellison et al., 2011) find that the median number of Facebook friends for college students is approximately 300, with the number of friends for some students exceeding 1,000 .
Adolescents rely on intimate relationships with peers to help them understand the desired behaviors for their cohort (Harter et al., 1996; Brechwald and Prinstein, 2011). Studies suggest that computer mediated communication can facilitate this intimacy by providing means for self-disclosure that helps adolescents maintain communication with close relations (Hampton et al., 2011), obtain emotional support (Ellison et al., 2007) and facilitates emotional closeness to their friends (Subrahmanyam et al., 2008). Mazer et al. (2007) was the first to recognize that Facebook possesses many features that promote self-disclosure such as posting photos, sharing 
thoughts, posting interests and linking to a broad social network spanning friends, colleagues and families.

Brechwald and Prinstein (2011) argue that adolescent development is not only based on adolescent modeling, but also on perceptions of social comparisons. In other words, adolescents are not only socialized by peer actions, but also by what they think their peers are doing. If adolescents believe that a peer is engaging in a specific behavior (even in the absence of any proof), then they are likely to engage in that behavior as well. Evidence for this is found in studies of misperception of alcohol use (Perkins et al., 2005) and peer's smoking behavior (Otten et al., 2009).

However with forms of electronic communication such as email, texting, Facebook and Twitter, adolescents have to decide what aspects of their personality to share and, therefore develop, in an increasing public forum. The complex effect of media sites such as Facebook on adolescent identity and behavior is compounded when considering that on these sites adolescents are "friends" with multiple people of different levels of personal closeness; yet, whenever they post something, it automatically is shared with all these people as if they were equally important to the discloser (Brandtzaeg et al., 2010). Consequently, comments that are aimed at a few may essentially be read by hundreds, which blurs the distinction between mass and interpersonal communication where one misstep can have very profound social, personal and professional consequences (Mesch and Talmud, 2006; Hinduja and Patchin, 2008; Walther et al., 2008).

Marwick (2012) notes that people who participate in mediated communities are highly aware that not only are they watching others, but that they are being watched as well Marwick uses the term "social surveillance" to refer to disclosers' awareness they are broadcasting information that other individuals will look at while they are simultaneously monitoring others. Since, according to Marwick, social media users are concerned with what their extended social networks, such as parents and bosses, may see, they self-monitor what they disclose online in order to maintain a balance between privacy and publicity. Furthermore, (Brandtzaeg et al., 2010) in a study of 16-62 year old Facebook users found that there is the concern of sharing too much, where people may strain existing networks by sharing too much personal information and, therefore, they strive to only share information that is not too private or personal.

On-line social networks provide adolescents with time to reflect and consciously choose the specific aspects of their identities that they want others to see
(Walther, 1996; Krasnova et al., 2010). Birnbaum (2013) notes that undergraduates consciously think about the impressions they want to make to peers on media sites like Facebook (Martinez-Alemán and Wartman, 2008); therefore, they spend a lot of time managing their profiles to provide the impression of themselves that they desire (Reich, 2010). This impression management is directly relevant to depictions of college drinking on Facebook. Many college students believe that college is a place to party and drink alcohol (Lo, 2000; Marciszewski, 2006) and students tend to think that participation in these behaviors are necessary to be socially accepted in college (Lo, 2000; Shinew and Parry, 2005). This is supported by (Peluchette and Karl, 2007) who studied 200 Facebook profiles and found that $42 \%$ had comments about alcohol and $53 \%$ had photos about alcohol use. The same study looked at what people posted on each other's profiles and found that $50 \%$ of the posts involved partying. Hence, Facebook profiles would contain posts and images that students intentionally believe are most likely to convince their audience, especially their peeers, that they are fitting into the expectations of college life (e.g., drinking), even if the discloser does not actually participate in these behaviors as much as the profile would suggest (Birnbaum, 2013). Furthermore, not only do college students need to make it look like they eagerly and willingly participate in this college partying culture, they need to do it in an "extreme" way that is viewed by this generation as "cool"; and, documenting this behavior on Facebook is a means of proof. Therefore, Facebook postings, according to (Birnbaum, 2013), are stylized performances of individuals wanting the approval of their peers. However, where the line between "cool" and negatively excessive lies is unclear. No studies have addressed whether peers perceive limits to appropriate Facebook depictions of college drinking or if they express disapproval to peers who are exceeding these limits.

Much of the research on undergraduates' use of Facebook is quantitative and atheoretical (Birnbaum, 2013); however, (Krasnova et al., 2010) applied Privacy Calculus Theory, an offshoot of social exchange theory, to establish a theoretical foundation of self-disclosure. According to social exchange theory, interpersonal relationships are based on a subjective weighing of benefits and costs of the relationship (Homans, 1958). Privacy Calculus Theory applies elements of social exchange theory to mediated communication and argues that users feel that the social return of self-disclosure offsets the risk of the potential compromise of privacy. Or to put it another way, the benefits of the trust 
building, mutual empathy, reciprocation of selfdisclosure and social acceptance outweigh the potential costs of having one's privacy be compromised (Joinson and Paine, 2007). Krasnova and colleague's findings support Privacy Calculus Theory because participants engaged in a conscious evaluation of the pros and cons of self-disclosure. If participants perceived a privacy risk (a con) they said that they consciously limited the amount and content of their disclosure.

When applied to the context of college students' Facebook depictions of alcohol consumption, Privacy Calculus Theory suggests that the benefits of showing peers that you fit into the college culture would outweigh the risk of privacy. However, existing theory and research do not examine the reviewer consequences that would help college students actually evaluate that risk. In other words, for college students to accurately weigh the pros and cons of privacy and disclosure, students need peer feedback for their disclosures.

For this process to work, it is implied that peers are giving not only positive feedback, but negative feedback for behaviors that move against these shared norms. Much research has been devoted to what adolescents are sharing on social network sites, but little research exists about what types or reactions they are getting to what they share. If their peers are not reacting negatively to inappropriate content, then they are tacitly giving the impression of support for that content-whether or not they really do support it. Therefore, if college students see extreme posts of alcoholic behavior without any negative feedback to show that these depictions are negatively received or perceived, consistent with Privacy Calculus Theory, college students may be tempted to copy this behavior due to the perception of low risk because this is what "cool" people are doing.

As mentioned earlier, most studies focus on the motivation of the discloser to reveal private information; little research exists regarding people's reactions to the information being disclosed. Studies of analogous avenues for public self-disclosure, such as media disclosures in talk shows where talk show guests share intimate information normally reserved for extremely close friends with millions of strangers, has been termed "public intimacies" by (Priest, 1995). Orrego et al., (2000) note, however, that such sharing of intimacies in such a large public venue is frequently seen as inappropriate and has become negatively associated with talk shows which frequently feature marginalized groups and inappropriate behavior as their means of "entertainment". Bazarova (2012) is one of the few researchers who studied people's reactions to disclosure on Facebook. Bazarova found that high intimacy disclosures were deemed less appropriate in public settings like a wall post than in more private settings such as personal messaging. Bazarova also found that this negatively affected the receiver's view of the discloser. In other words, receivers did not like disclosers who posted intimate issues in public as much as they liked disclosers who reserved these posting to more private venues. Even Bazarova's study, however, did not address specific aspects of posts or sharing that those students found distasteful, nor did the study address receiver's actions towards disclosers who posted negatively perceived high intimacy disclosures. Currently research addressing how receivers sanction individuals who post negatively perceived information on Facebook is surprisingly absent, especially since the reaction of others is an important part of adolescent socialization.

This study is an early attempt to fill this gap in the literature with a qualitative exploration of what college students consider to be inappropriate depictions of alcohol consumption on Facebook. This study also examines what, if anything, college students do to sanction their peers who cross this unspoken line. There is much research suggesting that adolescents use social media as a form of socialization, learning appropriate and inappropriate behavior through the feedback of others (Petronio, 2002). Adolescents today are very aware that others can view the material that they post on social media like Facebook, yet there are conflicting interests between the desire for peer acceptance and the concern over non-peer approval of material that is posted on Facebook. Many students believe that drinking alcohol is part of the collegiate experience and culture and showing that one consumes alcohol is an important part of maintaining/establishing that one fits into this atmosphere. On the other hand, college students are aware that others, such as family and potential employers, may see Facebook profiles and interpret alcohol consumption negatively. Therefore, the question remains, what do college students find appropriate and inappropriate in maintaining a balance between these competing forces and why. Furthermore, it is also unclear what college students do when they see their peers cross this line of appropriateness. In other words, what do college students do to let their peers know that online social behavior is inappropriate-or to put it another way, how do receivers contribute to discloser's socialization. 


\section{MATERIAL AND METHODS}

This data analyzed here is part of a larger focus group study at a small private liberal arts college in Pennsylvania. Students were offered extra credit to participate in a focus group regarding what they felt was and was not appropriate to post on Facebook for a variety of topics. Participants were placed into one of seven focus groups. Each focus group lasted approximately 45-70 min and consisted of between 6-8 students. All focus group discussions were recorded and transcribed. This article focuses only on those discussions that relate to alcohol consumption.

Because this research is a focus group design, instead of formulating hypotheses, the issues regarding perception of drinking posts were organized into two broad research questions in order to capture the meanings and actions students give to Facebook depictions. The research questions are:

- What behaviors regarding alcohol consumption do college students find to be inappropriate for selfdisclosure on Facebook and why

- When they see a peer disclosing alcohol related behavior in a perceived inappropriate way, how do they react to that post

In order to initiate discussions regarding appropriate posts for drinking alcohol, three scenarios of varying inappropriateness, all of which involved some form of self-disclosed drunk behavior, were manipulated. In all three instances, the posts began with the profile person (hereby called the "discloser") making a comment about looking forward to a party that night. Then there was a friend who later commented that she can't believe how drunk the discloser got that night. What was manipulated after that was the discloser's response to the comment made by the friend. The three possible responses, in order of increasing inappropriateness, are:

Mildly inappropriate (just a general acknowledgement of being drunk): "I know! I can't believe how drunk I got last night! Thank you BFFs for making sure I got home OK!".

Moderately inappropriate (admitting to vomiting in public): "I know! I can't believe I puked all over Ron's parents' expensive couch! Crazy!"

Highly inappropriate (praising others who also were extremely drunk): "What a party! I haven't puked so hard and so long from booze in a while! Here's too all of you who pushed your limits last night" Regardless of the manipulation, all three of the fake Facebook posts for alcohol consumption depicted people of the same gender (as indicated by a name and profile picture), similar geographical location, similar age (within a couple of months) and similar amount of Facebook friends. At the start of each focus group, students were given a short survey in order to obtain basic information regarding their demographics and their Facebook usage and to give them a place to rank the level of inappropriateness of each profile they viewed.

\section{RESULTS}

\subsection{Sample and Profile Characteristics}

Forty-six students total participated in the focus group discussions. The majority of the participants $(87 \%$, $\mathrm{n}=40$ ) currently used Facebook; and, of those users, $90 \%$ of them $(n=36)$ used Facebook at least once a day. All of the remaining students who were not currently using Facebook $(n=6)$ had previously used Facebook, but had stopped within the last 12-18 months for reasons such as job hunting, thinking it was "stupid", or a potential conflict with a university paid position (e.g., Resident Assistant). The students were evenly mixed between males and females (23 students each); and, although two students did not answer the question about academic year, the majority of students were juniors or seniors $(81.8 \%, \mathrm{n}=36)$. While all the students who have Facebook accounts claim that they have seen posts about drinking behavior, slightly more than half $(57.5 \%, \mathrm{n}=$ 23) claim that they have not posted anything about their own drinking behavior on Facebook.

\subsection{Research Question 1}

Two themes of inappropriateness emerged based on focus group discussions, as did two main reasons for those themes. The most evident theme focused on the degree of intoxication the discloser expressed. While many participants recognized that college students "party" and "drink alcohol", there was a strong sense that it was inappropriate to mention being physically sick in any manner on Facebook. Participants responded negatively in the focus groups to both the mention of vomiting on a parent's couch and to claiming to vomit so hard that one "pushed their limits". Even though students were more likely to rate the highest inappropriate post more negatively $($ mean $=4.38, \mathrm{~s}=$ 0.90 ) than the moderately inappropriate post (mean $=$ $4.0, \mathrm{~s}=0.92$ ), the general distaste for posting about drunken vomiting prevailed in both posts, although it 
was not mentioned at all when students discussed the mildly inappropriate post. All participants are identified by pseudonyms and the reference in parentheses refers to the post level of inappropriateness to which they are referring.

Kurt (medium): OK, she had fun. But clearly she took things too far in puking...she could have just said she went to a party and stopped there. She had to go say she puked so hard and for a long time.

Nolan (medium): I felt no trouble all the way up until what she said, that she puked. That is not something she should have put on there.

Karen (high): I think it's inappropriate in its content...but it's really disgusting. It's over the top as far as her actually talking about puking and stuff like that.

Addison (high): It's one of those things again. It was fine in the beginning, like it doesn't give much detail, but then you are talking about puking. That's just too much information.

It appears that sharing exploits about drunken vomiting falls under the category of "too much information". This supports (Bazarova, 2012) findings that not only are high intimacy posts on walls deemed inappropriate, but that they also negatively affect the receiver's view of the discloser. Some students commented that discussing vomiting simply makes the discloser look "stupid" or that it was a move for attention that was also viewed negatively.

The second theme of inappropriateness reflected an awareness of the discloser's expressed age. While the researcher did not draw attention to the discloser's personal characteristics, many of the participants noticed that the discloser was not of legal drinking age based on the birthdate that appeared on the profile. As a result, they felt that any posting of drunken behavior on Facebook for this person was generally inappropriate. This was noticed by participants in all three levels of profile drunkenness, suggesting that the age issue was a theme independent of how drunk the person appeared to be.

Addison (low): I noticed...that you can see her birthdate and she is not 21 . So I guess if you were like 21 , it would be more acceptable if you posted that because you are legally allowed to do that. But she is not legally allowed to drink, so it is really not something she should put on Facebook.

Cathy (medium): Looking at her age is kind of what helps me to distinguish that it's inappropriate. I just feel that being that young...(respondent trailed off) Nancy (high): She is obviously underage...I think it really makes her look irresponsible that she's talking about puking and she's proud of it.

When participants were asked whether the post they viewed would be appropriate if the discloser was of legal drinking age, for the low-inappropriate post, all responded that it would be. For example:

Kimberly (low): It's pretty normal. I don't usually comment on it. A lot of my friends are 21 or newly turning 21 so a lot of Facebook is about 21th birthday parties and stuff like that.

Danni (low): I think this is more normal for me to see on-line, so that's why I really don't find it that offensive. It also may be because I am a 21 year old female college student...to me it was fine because that's what I see a lot online.

However, for the next two more inappropriate posts, students still felt that these posts were inappropriate regardless of the age of the discloser.

Both reactions to the profiles and comments made in focus group discussion suggests that these students are generally tolerant and may even expect (as indicated by comments suggesting that drinking in college is "normal" or that they see a lot of posts among their peers who are 21) peers to drink alcohol. However, they do not feel that it is appropriate for people under the legal drinking age to post their drinking exploits on Facebook, nor do they find it appropriate for individuals to disclose that they vomited, regardless of the discloser's age or where the profile individual vomited. Interestingly, other aspects of vomiting, such as whether the person admitted to doing it privately or in public, did not appear in any of the focus group discussions. Students were offended by the mention of vomiting itself-the context in which the vomiting occurred was not nearly as relevant as the inappropriateness of mentioning it at all.

There were two themes explaining the age and vomiting perceptions. The first was the awareness of Facebook as a social medium that reaches a wide array of individuals. Students in these focus groups were very aware that the information that they post could be viewed by others-from family members to potential employers. Again, this concern for the opinion of others who were viewing the post transcended the degree of drunkenness and appeared across all three inappropriate thresholds.

Katie (low): I think it's inappropriate because she's talking about how drunk she got and sometimes employers can look on Facebook. 
Dany (medium): I have family on Facebook and I really wouldn't want them to know that I puked.

Andrew (high): Employers can see stuff on your wall. If an employer saw that, I am pretty sure they wouldn't hire you. Especially since it says she was born in 1995 .

These students recognize that social network sites like Facebook involve many "friends" with whom the discloser has varying degrees of closeness (Brandtzaeg et al., 2010). What may be appropriate among college friends may not be appropriate among family or potential employers.

College student are likely to post comments about excessive drinking on Facebook, even with awareness that others might see the posts (Birnbaum, 2013). However, these students definitely recognized a level that was "too much" sharing and vomiting crossed that level. The second reason that the mention of vomiting was considered to be inappropriate is because the information shared was deemed as unnecessary or "too much".

Warren (medium): Well, who wants to read that? I don't think anybody really cares. I don't care in the least.

Thomas (medium): There's no need to talk about it, like there really isn't. If you were drunk, then you had a good time with your friends, that's it. There's literally no purpose for you to put this on Facebook at all.

Jim (high): You don't need to tell the whole world that you are drinking or how drunk you got. I don't think that that's necessary.

Anthony (high): There are more tactful ways of saying you had a good time.

When asked if they saw posts about vomiting on Facebook, the students in this sample overwhelmingly admitted that they did and that their reactions were similar to the posts presented for discussion.

\subsection{Research Question 2}

The second broad research question asked these students what they did when they saw posts involving college drinking that they felt were inappropriate. Overwhelmingly these students responded that they "ignored" the posts or just "scrolled past it", with little or no more clarification. In fact, these students seemed so indifferent to the posts that they did not merit any additional attention on their part at all.

Tony (low): I generally ignore it. I don't really care.

Carter (medium): I would just ignore it and say like "Oh, wow. There goes another weekend."

Kurt (medium): I would just scroll past it.

None of the 46 students said that they would actually post a negative comment on Facebook about these posts or even text the discloser about the inappropriateness.
Only a couple of participants said that they would delete the individual who made these posts and only then if posts about drinking excessively was "all that they wrote about". These ambivalent responses to posts that students claim to find inappropriate questions how strongly, or at leat in what manner, peers are acting as socializing agents on Facebook. If through interpersonal conversations, peers develop shared norms and create a sense of belonging with like-minded people (Arnett, 1996; Rubin et al., 2006; Brechwald and Prinstein, 2011) and Facebook is a form of mass communication, it is implied that peers are giving not only positive feedback, but negative feedback for behaviors that move against these shared norms. If peers are not reacting negatively to inappropriate content, then they are tacitly giving the impression of support for that content-whether or not they really do support it.

When asked why they would not and in the case of real life did not, comment on inappropriate posts, students often claimed that they did not care enough about the post or that, while the post was inappropriate, it was also somewhat "entertaining."

Carter (medium): I find it entertaining....They just post ridiculous stuff that is entertaining.

Jim (high): I ignore it because...it's ridiculous stuff and it's kind of entertaining.

Kurt (medium: It is entertaining to see what people post. Like the dumb things they say.

This entertainment reaction is consistent with Orrego et al., (2000) findings that people observe inappropriate behavior as a means of entertainment. However, these students' responses do question the direction of the socializating influence. In other words, by not correcting perceived inappropriate behavior and even noting some of it as entertaining, receivers may unintentionally give the impression of acceptance which would encourage the discloser to continue the behavior, not correct it.

\section{DISCUSSION}

Much research exists examining adolescent peer socialization and self-disclosure on social network sites such as Facebook. However, many of these studies are quantitative and they generally focus on the decisions of disclosure on behalf of the individual disclosing. There are few studies that are qualitative and examine the perception of receivers on Facebook posts-especially with regards to what they specifically define as inappropriate behavior and what, if any, reactions receivers will give to disclosers for negative postings. To 
address this gap, focus groups of college students were conducted to examine their view of appropriateness for a variety of social behaviors. This study pertains to the discussions surrounding the consumption of alcohol and specifically addressed two research questions. The first research question focused on what behaviors regarding alcohol consumption college students find to be inappropriate for self-disclosure on Facebook and why. The second was to see how college students reacted when they saw people disclose behavior on Facebook that they felt was inappropriate.

The findings suggest that the students in these focus groups are not necessarily against the consumption of alcohol in college, or against the posting of such behavior in general on Facebook. However, consistent with Privacy Calculus Theory, they do have fairly consistent rules and reasons for how to disclose this behavior appropriately. Many of these students felt that if a discloser was under the age to legally drink, even a general posting on Facebook about being drunk was inappropriate. The main reason for the age theme was because many of these students felt that Facebook friends may include family members or they were concerned about employers who might use Facebook when checking applications. These students felt that the potential cost of admitting to illegal behavior to family and (possibly) employers would outweigh any benefit of fitting in with peers.

These students also felt that any reference to public vomiting was inappropriate. These students found this type of information to be too much and/or unnecessary and expressed wonder as to why someone would think that someone else would need to know that. Expressions of "too much" and "[I did] not need to know that", in reactions to hearing about vomiting, even though the students were generally ok with the mention of being drunk or at a party (as long as the discloser was of legal drinking age), suggests that these students recognize the pros of participating in the college culture, but also identify costs of too much information, which also supports Privacy Calculus Theory. Here as well they expressed concern over what type of impression that the posting of such behavior would give to family or employers.

Nonetheless, even though these students found these behaviors to be inappropriate, the majority admitted to having Facebook "friends" who posted similar behavior. One has to wonder then why students would post such information, especially since on-line social networks provide adolescents with time to reflect and consciously choose the specific aspects of their identities that they want others to see (Walther, 1996; Martinez-Alemán and Wartman, 2008; Krasnova et al., 2010;). Because college is still seen as a place to party and drink alcohol, not only do college students need to make it look like they eagerly and willingly participate in this college partying culture, they need to it in an "extreme" way that is viewed by this generation as "cool" (Birnbaum, 2013). The problem is, these findings suggest that students do not find this behavior to be "cool"; however, by their own admission, they do not express any negative sanctions to disclosers when they exhibit this behavior. In fact, the students in this sample were unlikely to express any reaction at all. They find the posts to be inappropriate, but sometimes entertaining and generally not worth their time to comment, even if they know the person well. They also admit to thinking negatively about peers who post these behaviors. This would suggest limitations to Privacy Calculus Theory because receivers are not providing negative consequences that would accurately help disclosers evaluate the risk of those types of posts. This absence of negative feedback may indirectly reinforce the discloser's sense of social acceptance (Reich, 2010) even though the reviewer's reaction is the opposite. Even among students who mentioned that these posts are "entertaining", the implication was that the entertainment was in a negative direction, much like some find the sharing of public intimacies among marginalized groups to be entertaining (Orrego et al., 2000). Either way, these focus groups suggest that negative sanctioning is not occurring; therefore, the misperception that posting on Facebook about excessive alcohol use and underage drinking are socially desirable are likely to persist.

Although this study provides some useful insights into what college students consider to be acceptable to disclose about alcohol consumption on Facebook and suggests that they ignore, as opposed to negatively sanction, such behavior, it is only an early step. There are a number of limitations that prevent this study from being generalized to a wider college population. Given that this was a focus group design conducted at one small, private college with a relatively small sample size, these findings cannot be generalized to other schools or to a larger population. The strong themes, however, do suggest merit in conducting further analysis on a larger, more diverse population. There is also the possibility that the scenarios that were designed to stimulate discussion may have inadvertently limited discussion by limiting the scope of potentially negative alcoholic depictions. The only aspect of drunken behavior manipulated in the scenarios was whether one vomited and how the 
discloser described/handled the situation. Based on the posted birthday, students noticed that all of the disclosers were under the legal drinking age. However, even in general discussion, no one else mentioned any behaviors beyond vomiting and age that were inappropriate to post about. It may be that the information they noted on the profiles limited what they considered to be inappropriate to these two dimensions. There may be other aspects of alcoholic consumption, such as posting about drunken fights or drunken hookups that students feel should not be posted on Facebook, but because they were focused on the scenarios, they did not consider them. Last, the majority of this sample was upperclassmen. A more diverse population may lead to different concerns and reasons for inappropriateness. For example, upperclassmen may be more worried about employers seeing material than underclassmen may be.

\section{CONCLUSION}

One strength of the focus group design is that it can provide a rich description of subjective understanding of a phenomenon and this description is limited in the literature (Birnbaum, 2013). While some studies suggest that college students perceive excessive drinking in college to be important to showing peers that they are part of a "cool" college culture, the students in this sample found Facebook posts boasting of underage drinking and vomiting to be inappropriate. If future research can establish this link more quantitatively, then this could be an important tool when educating students both about campus life and about how to depict themselves on social media sites like Facebook. Furthermore, even though college students may not necessarily directly negatively sanction peers who make such posts, it is important to let adolescents know that just because they do not get negative feedback from friends on Facebook, does not mean that their friends are agreeing with or supporting a behavior. Friends may be laughing at the discloser, not with him or her. This information, if supported by a larger more quantitative study, can be used to disassemble student perceptions of what their peers think is "cool" college behavior; as well as further enlightening them about the importance of what they post on social media sites.

\section{ACKNOWLEDGMENT}

This author was financed by the University of Scranton. The author is thankful to the university and to the students who participated in the focus groups.

\section{REFERENCES}

Arnett, J.J., 1996. Metalheads: Heavy Metal Music and Adolescent Alienation. 1st Edn., Illustrated, Westview Press, Boulder, ISBN-10: 0813328136, pp: 196.

Bazarova, N., 2012. Public intimacy: Disclosure interpretation and social judgments on Facebook. J. Commun., 62: 815-832. DOI:10.1111/j.14602466.2012.01665.x

Birnbaum, M., 2013. The fronts students use: Facebook and the standardization of selfpresentations. J. College Student Dev., 54: 155-171. DOI: $10.1353 /$ csd.2013.0022

Brandtzaeg, P., M. Lüders and J. Skjetne, 2010. Too many Facebook "friends" content sharing and sociability versus the need for privacy in social network sites. Int. J. Human-Comput. Interact., 26: 1006-1030. DOI: 0.1080/10447318.2010.516719

Brechwald, W. and M. Prinstein, 2011. Beyond homophily: A decade of advances in under-standing peer influence processes. J. Res. Adolescence, 21: 166-179. DOI: 10.1111/j.1532-7795.2010.00721.x

Brenner, J., 2012. Pew Internet: Social networking (full detail). Washington, Pew Res. Center's Int. Am. Life Proj.

Ellison, N.B., C. Steinfield and C. Lampe, 2007. The benefits of Facebook "friends:" Social capital and college students' use of online social network sites. J. Comput. Med. Commun., 12: 1143-1168. DOI: 10.1111/j.1083-6101.2007.00367.x

Ellison, N.B., C. Steinfield and C. Lampe, 2011. Connection strategies: Social capital implications of facebook-enabled communication practices. New Media Society, 13: 873-892. DOI: 10.1177/1461444810385389

Hampton, K.N., L.S. Goulet, L. Rainie and K. Purcell, 2011. Social networking sites and our lives: How people's trust, personal relationships and civic and political involvement are connected to their use of social networking sites and other technologies. WorldCat.

Harter, S., C. Stocker and N. Robinson, 1996. The perceived directionality of the link between approval and self-worth: The liabilities of a looking glass. Self-orientation among young adolescents. Dev. Personality Self Ego Adolescence, 3: 285-308.

Hinduja, S. and J. Patchin, 2008. Personal information of adolescents on the internet: A quantitative content analysis of MySpace. J. Adolescence, 31: 125-146. DOI: 10.1016/j.adolescence.2007.05.004 
Homans, G.C., 1958. Social behavior as exchange. Am. J. Sociol., 63: 597-606.

Joinson, A.N. and C.B. Paine, 2007. Self-disclosure, privacy and the Internet. In: Oxford Handbook of Internet Psychology. Joinson, A.N., K. McKenna, T. Postmes and U. Reips (Eds.)., Oxford University Press, ISBN-10: 0198568002, pp: 237-252.

Krasnova, H., S. Spiekermann, K. Koroleva and T. Hildebrand, 2010. Online social networks: Why we disclose. J. Inform. Technol., 25: 109-125. DOI: DOI: $10.1057 /$ jit.2010.6

Lo, C.C., 2000. The impact of first drinking and differential association on collegiate drinking. Sociol. Focus, 33: 265-280.

Marciszewski, A., 2006. Peer persuasion: Universities turn to students to help battle alcohol abuse. Tulsa World.

Martinez-Alemán, A.M. and K.L. Wartman, 2008. Online Social Networking on Campus: Understanding What Matters in Student Culture. 1st Edn., Routledge, New York, ISBN-10: 1135855277, pp: 168.

Marwick, A.E., 2012. The public domain: Social surveillance in everyday life. Surveillance Society, 9: 378-393.

Mazer, J., R. Murphy and C. Simonds, 2007. I'll see you on "Facebook": The effects of computer-mediated teacher self-disclosure on student motivation, affective learning and classroom climate. Commun. Edu., 56: 1-17. DOI: 10.1080/03634520601009710

Mesch, G. and I. Talmud, 2006. The quality of online and offline relationships: The role of multiplexity and duration of social relationships. Inform. Society, 22: 137-148. DOI: 10.1080/01972240600677805

Orrego, V.O., S.W. Smith, M.M. Mitchell, A.J. Johnson and B. Greenberg et al., 2000. Disclosure and Privacy Issues on Television Talk Shows. In: Balancing the Secrets of Private Disclosures. Petronio, S. (Ed.)., Lawrence Erlbaum Associates, Mahwah, ISBN-10: 0805831142, pp: 249-259.

Otten, R., R.C.M.E. Engels and M.J. Prinstein, 2009. A prospective study of perception in adolescent smoking. J. Adolescent Health, 44: 478-484. DOI: 10.1016/j.jadohealth.2008.09.004

Peluchette, J. V. and K.A. Karl, 2007. The prevalence of facebook faux pas and students' "devil may care" attitudes. Proceedings of the Midwest Academy of Management Meeting, Oct. 4-6, Kansas City, Missouri.
Perkins, H.W., M.P. Haines and R. Rice, 2005. Misperceiving the college drinking norm and related problems: A nationwide study of exposure to prevention information, perceived norms and student alcohol misuse. J. Studies Alcohol, 66: 470478. PMID: 16240554.

Petronio, S., 2002. Boundaries of Privacy: Dialectics of Disclosure. 1st Edn., State University of New York Press, Albany, ISBN-10: 0791455165, pp: 268.

Priest, P., 1995. Public Intimacies: Talk Show Participants and Tell-All TV. 1st Edn., Hampton Press, Cresskill, ISBN-10: 157273003X, pp: 246.

Reich, S.M., 2010. Adolescents' sense of community on myspace and facebook: A mixed-methods approach. J. Commun. Psychol., 38: 688-705. DOI: 10.1002/jcop.20389

Rubin, K., W. Damon, M. Richard, Lerner and N. Eisenberg, 2006. Peer Interactions, Relationships and Groups. In: Handbook of Child Psychology, Social, Emotional and Personality Development. Eisenberg, N. (Ed.),, John Wiley and Sons, ISBN-10: 0471756121, pp: 619-700.

Shinew, K.J. and D.C. Parry, 2005. Examining college students' participation in the leisure pursuits of drinking and illegal drug use. J. Leisure Res., 37: 364-387.

Subrahmanyam, K., S.M. Reich, N. Waechter and G. Espinoza, 2008. Online and offline social networks: Use of social networking sites by emerging adults. J. Applied Dev. Psychol., 29: 420-433. DOI: 10.1016/j.appdev.2008.07.003

Walther, J.B., B.V.D. Heide, S.Y. Kim, D. Westerman and S.T. Tong, 2008. The role of friends' appearance and behavior on evaluations of individuals on Facebook: Are we known by the company we keep. Human Commun. Res., 34: 2849. DOI: $10.1111 / \mathrm{j} .1468-2958.2007 .00312 . x$

Walther, J.B., 1996. Computer-mediated communication impersonal, interpersonal and hyperpersonal interaction. Commun. Res., 23: 3-43. DOI: 10.1177/009365096023001001 